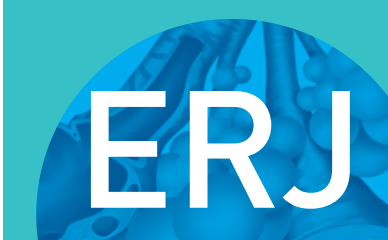

open research

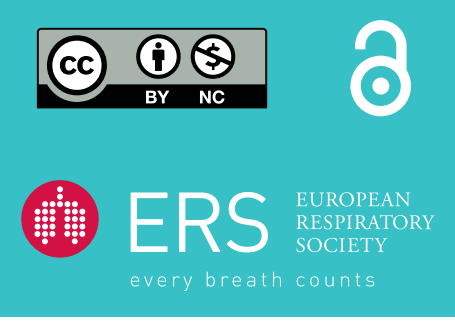

\section{Prolidase deficiency diagnosed by whole exome sequencing in a child with pulmonary capillaritis}

\section{To the Editor:}

Diffuse alveolar haemorrhage (DAH) is rare in the paediatric population and the biological mechanisms remain poorly understood [1]. We retrospectively studied 12 children at our centre, identified from our pathology database, with idiopathic DAH between 2005-2017 who had undergone lung biopsy (SickKids ethics approval number: 1000029185). Two children in this cohort were offered clinical whole exome sequencing (WES) as an investigational diagnostic procedure based on a family history of lung disease. Both children who underwent WES, including the one presented here and one who we had previously reported [2], had single gene mutations that explained the pathogenesis of their pulmonary haemorrhage.

A 16-month-old boy of South Asian descent with a history of repaired cleft lip and palate presented to our institution with a sub-acute history of pallor and fatigue, severe anaemia (haemoglobin $33 \mathrm{~g} \cdot \mathrm{L}^{-1}$ ) and diffuse interstitial markings on chest radiograph. An unenhanced computed tomography (CT) scan of the chest revealed diffuse, patchy ground-glass opacities, interlobular septal thickening and tiny subpleural lucencies (figure 1a and b). Bronchoalveolar lavage identified DAH (progressively bloody returns, haemosiderin-laden macrophages). Autoantibody screen was abnormal (anti-nuclear antibody 1:320, anti-neutrophil cytoplasmic antibody (ANCA) 1:320, anti-myeloperoxidase $30 \mathrm{U} \cdot \mathrm{mL}^{-1}$ (positive)). Open lung biopsy revealed recent and old intra-alveolar haemorrhage, characterised by accumulation of haemosiderin-laden macrophages in alveolar spaces, haemosiderin incrustation of the walls of small vessels and alveolar septa as well as mild, focal neutrophilic pulmonary capillaritis (figure 1e). In addition, there were foci of lipoid pneumonia (figure 1e). Electron microscopy findings were consistent with changes seen in pulmonary haemosiderosis or capillaritis, with no immune complex deposition (figure 1f) [1].

The boy was diagnosed with ANCA-associated pulmonary capillaritis. After successful induction therapy with high dose prednisone and rituximab, he was maintained on a low dose of oral prednisone and rituximab infusions for 24 months. His rituximab dose was titrated based on his clinical symptoms, autoantibody titres, CD-19 positive lymphocyte counts and peripheral reticulocytosis. On 3-monthly rituximab, he had no clinical evidence of haemorrhage and good suppression of his ANCA titres and CD19 counts; however, there was a persistent non-clonal gammopathy (serum IgG concentration between 15-20 g. $\left.\mathrm{L}^{-1}\right)$. Despite his apparent good clinical disease control, a CT scan of the chest at the age of 5 years revealed progressive pulmonary fibrosis and cystic changes (figure 1c and d).

Family history was significant for idiopathic pulmonary capillaritis in the young child of a consanguineous union of paternal second cousins. While there was no reported consanguinity in our index case and clinical assessment did not reveal any phenotypically identifiable genetic syndrome, WES was pursued because of the family history of lung disease. WES revealed a novel homozygous missense variant in PEPD (NM_000285.3: c.1244T>A (p.Ile415Asn)). Parents were both found to be heterozygous for the same $P E P D$ variant, however extended family members outside of the index family were unavailable for testing. The PEPD variant is extremely rare in overall (0.0076\%) and South Asian (0.054\%) control populations [3], and in silico programs predict it to be damaging. Based on this result, a diagnosis of prolidase deficiency

@ERSpublications

The case of a young boy with pulmonary haemorrhage who was ultimately diagnosed on whole exome sequencing with a rare condition called prolidase deficiency. This case demonstrates the utility of modern genomic testing in paediatric rare lung disease. http://ow.ly/rDGz30o8pcd

Cite this article as: Rayment $\mathrm{JH}$, Jobling R, Bowdin S, et al. Prolidase deficiency diagnosed by whole exome sequencing in a child with pulmonary capillaritis. ERJ Open Res 2019; 5: 00205-2018 [https://doi.org/10.1183/23120541.00205-2018].

Copyright @ERS 2019. This article is open access and distributed under the terms of the Creative Commons Attribution Non-Commercial Licence 4.0. 


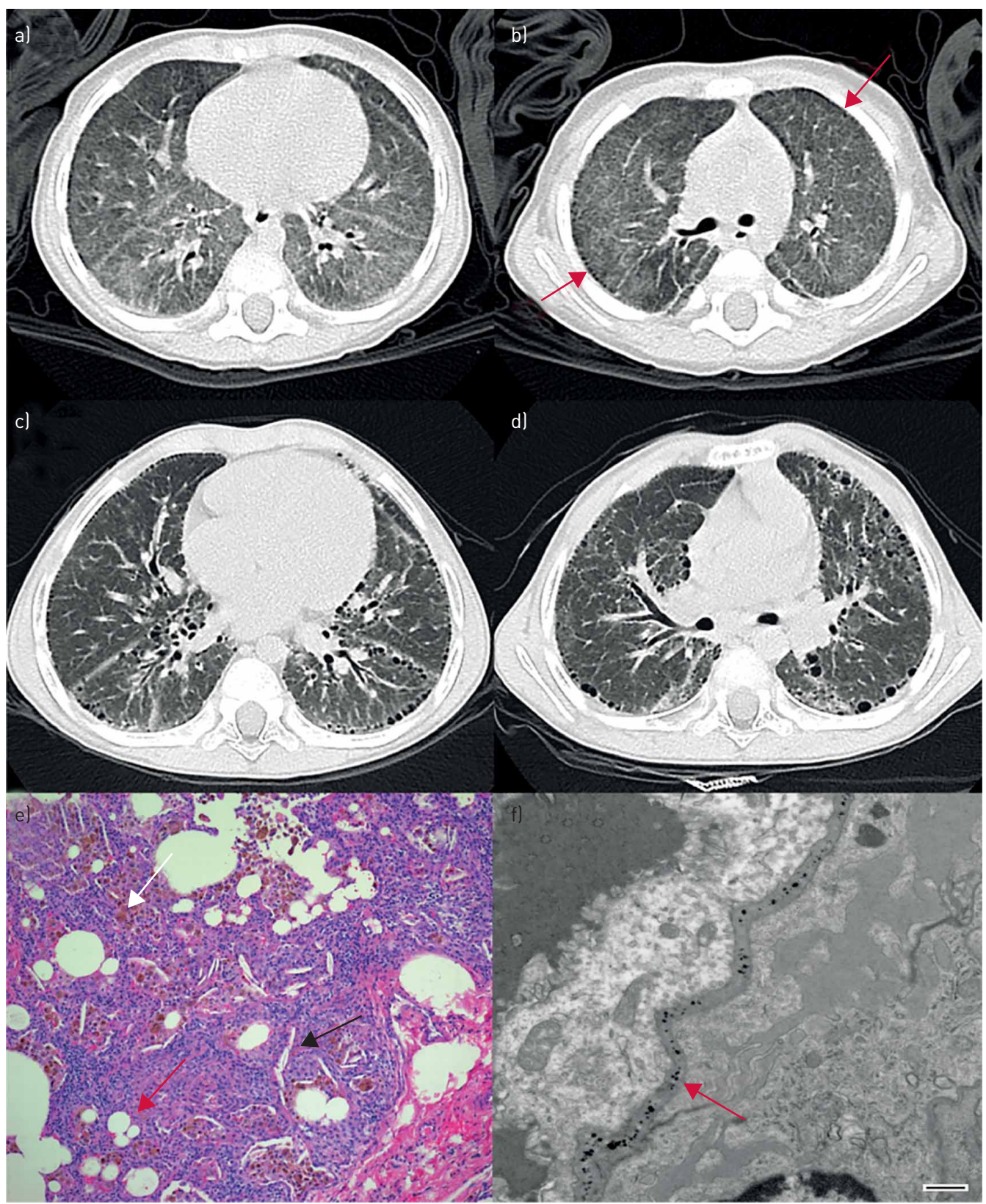

FIGURE 1 Radiology and histopathology. a, b) Computed tomography (CT) of the chest taken at 16 months of age at presentation of idiopathic diffuse alveolar haemorrhage, demonstrating diffuse ground-glass opacities, interlobular septal thickening and tiny subpleural cystic lesions (arrows). c, d) CT of the chest taken at 5 years of age, demonstrating significant resolution of ground-glass opacities after immunosuppressive therapy, but progression of the interlobular septal thickening and subpleural cysts (now also within a fissural and peribronchovascular distribution), with emergent traction bronchiectasis and fibrosis. e) Histopathology from the biopsy taken at 16 months of age demonstrating lipoid pneumonia (red arrow), cholesterol crystals (black arrow) and hemosiderin-laden macrophages (white arrow); haematoxylin and eosin stain, magnification 4x. f) Electron microscopy shows capillary basement membrane with electron dense mineral deposits (iron calcium phosphate, dark deposits seen along the length of basement membrane, indicated by the red arrow). Scale bar $=200 \mathrm{~nm}$.

was suspected. The diagnosis was confirmed with urine amino acid analysis [4], which showed imidodipeptiduria.

24 months after the discontinuation of all immunosuppressive medications, the patient has had no acute flare ups of his disease, his haemoglobin remains within the normal limits, his first pulmonary function tests are normal (forced vital capacity $91 \%$ predicted; forced expiratory volume in $1 \mathrm{~s} 90 \%$ predicted) and he is normoxic in ambient air, although his ANCA autoantibodies have returned. 
Prolidase deficiency is a rare (1-2 per 1000000$)$, autosomal recessive metabolic disease that results from a loss of function of the prolidase enzyme, encoded by $P E P D$, which is a dipeptidase that cleaves (hydroxy) proline-containing dipeptides. This results in an accumulation of these dipeptides in body tissues. Diagnosis is confirmed by the presence of imidodipeptiduria or using ex vivo peptidase assay. Clinically, this disease has variable severity, with multisystem involvement manifesting as developmental disability, chronic cutaneous ulceration, recurrent respiratory infections, chronic lung disease, hypergammaglobulinaemia, cytopenias and a typical facial dysmorphology $[5,6]$. There is a well-described association between systemic lupus erythematosus (SLE) and prolidase deficiency with $\sim 14 \%$ of patients with this condition reported to have clinical features of SLE [7]. The mechanism of this autoimmunity is unclear, but impaired proteolytic cleavage of immune modulators has been hypothesised to play a role [8]. Patients with SLE can also present with pulmonary haemosiderosis, associated with immune complex deposits in the basement membrane of alveolar capillaries [1]. However, there was no immune complex deposition observed on electron microscopy in this case (figure 1f).

The pulmonary manifestations of prolidase deficiency have been recently reported [9]. Clinically, a progressive obstructive defect and recurrent pulmonary infections have been observed. Interestingly, there are no cases of ANCA-positive pulmonary haemorrhage or capillaritis associated with prolidase deficiency reported in the literature. Radiographically, the subpleural cystic changes and ground-glass opacifications previously described [9] are strikingly similar to the progressive findings in our case. In addition, our patient showed radiographic evidence of progressive pulmonary fibrosis and interlobular septal thickening, which has also not been previously described. This element of fibrosis may be secondary to prolonged chronic, subclinical DAH in our patient. Whether the cystic changes are due to a pulmonary developmental abnormality or chronic haemorrhage is not clear, although the lung biopsy showed no evidence of an alveolar growth abnormality. The precise mechanisms of intra-alveolar haemorrhage and chronic lung disease in prolidase deficiency are currently unknown, although inefficient collagen recycling, necrosis-like cell death and oxidative stress may play a role [9]. This could also theoretically disrupt the alveolar capillary membrane, predisposing to alveolar haemorrhage. We suggest that prolidase deficiency should be added to the growing list of differential diagnoses underlying DAH [1].

This case demonstrates the utility of modern genomic testing in the setting of a rare lung disease, as recommended by the 2015 Pediatric Rare Lung Disease NIH-NHLBI Workshop [10]. To our knowledge, this is the first description of ANCA-associated DAH in prolidase deficiency. In this case, the diagnosis of prolidase deficiency would probably not have been made without WES analysis, and this association would have gone unrecognised. In addition to typical imaging and biopsy workup, we suggest that paediatric respirologists should consider genomic testing, especially when there is a positive family history of rare lung disease or consanguinity. The future concomitant acquisition of genetic and pathological data will improve our understanding of disease pathogenesis, will allow clinicians to arrive at more precise and timely diagnoses, and will facilitate the path to development of effective treatment options for children with rare lung disease.

Jonathan H. Rayment $\mathbb{\oplus}^{1,2}$, Rebekah Jobling ${ }^{3,4}$, Sarah Bowdin ${ }^{5}$, Ernest Cutz ${ }^{6}$ and Sharon D. Dell ${ }^{3,7,8,9}$ ${ }^{1}$ Division of Respiratory Medicine, BC Children's Hospital, Vancouver, BC, Canada. ${ }^{2}$ Dept of Paediatrics, University of British Columbia, Vancouver, BC, Canada. ${ }^{3}$ Dept of Paediatrics, University of Toronto, Toronto, ON, Canada. ${ }^{4}$ Division of Clinical and Metabolic Genetics, The Hospital for Sick Children, Toronto, ON, Canada. ${ }^{5}$ Dept of Clinical Genetics, Addenbrooke's Treatment Centre, Cambridge University Hospitals, Cambridge, UK. ${ }^{6}$ Division of Pathology, Dept of Paediatric Laboratory Medicine, The Hospital for Sick Children, Toronto, ON, Canada. ${ }^{7}$ Division of Respiratory Medicine, The Hospital for Sick Children, Toronto, ON, Canada. ${ }^{8}$ Institute of Health Policy, Management and Education, University of Toronto, Toronto, ON, Canada. ${ }^{9}$ Child Health Evaluative Sciences, The Hospital for Sick Children Research Institute, Toronto, ON, Canada.

Correspondence: Sharon D. Dell, Division of Respiratory Medicine, Room 4543, The Hospital for Sick Children, 555 University Ave, Toronto, ON, M5G 1X8, Canada. E-mail: sharon.dell@sickkids.ca

Received: Nov 022018 | Accepted after revision: Feb 282019

Prior abstract publication/presentation: This case was presented at the 2018 Conference of the American Thoracic Society: Rayment J, Bowdin S, Cutz E, et al. Prolidase deficiency and ANCA-associated vasculitis in a 16-month old boy. Am J Respir Crit Care Med 2018; 197: A5655.

Conflict of interest: None declared. 


\section{References}

1 Viero S, Cutz E. Pulmonary haemosiderosis. In: David TJ, ed. Recent Advances in Paediatrics. 19th Edn. New York, Churchill Livingstone, 2001.

2 Watkin LB, Jessen B, Wiszniewski W, et al. COPA mutations impair ER-Golgi transport and cause hereditary autoimmune-mediated lung disease and arthritis. Nat Genet 2015; 47: 654-660.

3 Lek M, Karczewski KJ, Minikel EV, et al. Analysis of protein-coding genetic variation in 60,706 humans. Nature 2016; 536: 285-291.

4 Ferreira CR, Cusmano-Ozog K. Spurious elevation of multiple urine amino acids by ion-exchange chromatography in patients with prolidase deficiency. JIMD Rep 2017; 31: 45-49.

5 Ferreira C, Wang H. Prolidase Deficiency. In: Pagon RA, Adam MP, Ardinger HH, et al., eds. GeneReviews(R). Seattle, WA, 1993

6 Falik-Zaccai TC, Khayat M, Luder A, et al. A broad spectrum of developmental delay in a large cohort of prolidase deficiency patients demonstrates marked interfamilial and intrafamilial phenotypic variability. Am J Med Genet $B$ Neuropsychiatr Genet 2010; 153B: 46-56.

7 Butbul Aviel Y, Mandel H, Avitan Hersh E, et al. Prolidase deficiency associated with systemic lupus erythematosus (SLE): single site experience and literature review. Paediatr Rheumatol Online J 2012; 10: 18 .

8 Kurien BT, D'Sousa A, Bruner BF, et al. Prolidase deficiency breaks tolerance to lupus-associated antigens. Int J Rheum Dis 2013; 16: 674-680.

9 Nir V, Ilivitky A, Hakim F, et al. Pulmonary manifestations of prolidase deficiency. Paediatr Pulmonol 2016; 51: 1229-1233.

10 Young LR, Trapnell BC, Mandl KD, et al. Accelerating Scientific Advancement for Paediatric Rare Lung Disease Research. Report from a National Institutes of Health-NHLBI Workshop, September 3 and 4, 2015. Ann Am Thorac Soc 2016; 13: 385-393. 